\title{
Nuclide Guide and International Chart of Nuclides - 2006
}

\author{
T. Golashvili ${ }^{1}$, , S. Badikov ${ }^{1}$, V. Chechev ${ }^{2}$, Huang Xiaolong ${ }^{3}$, Ge Zhigang ${ }^{3}$, and Wu Zhendong ${ }^{3}$ \\ 1 Central Research Institute of Management, Economics and Information (Atominform), 127434 Moscow, Russia \\ 2 V.G. Khlopin Radium Institute, 194021 St. Petersburg, Russia \\ 3 China Institute of Atomic Energy, 102413 Beijing, China
}

\begin{abstract}
New versions of the Nuclide Guide and the Chart of Nuclides were developed as a result of RussianChinese collaboration. The Nuclide Guide contains the basic information on more than 3000 radioactive and stable nuclides. The characteristics of isomers with half-lives more than $1 \mathrm{~ms}$ are included. For each nuclide spin, parity, mass of nuclide, magnetic moment (if available), mass excess, half-life or abundance, decay modes, branching ratios, emitted particles, energies of most intense gamma-rays and their intensities, decay energies and mean values of radiation energy per decay are given. For stable and natural long-lived nuclides cross-sections of thermal neutron induced activation are indicated. The information presented in the Guide was compiled from 5 sources: 1) ENSDF2006, 2) atomic mass evaluation-2003 by Audi and Wapstra, 3) interactive data bases at web-sites www.nndc.bnl.gov, www.nucleide.org, 4) original evaluations of authors, 5) recent publications. The International Chart of Nuclides was developed on the basis of information presented in Nuclide Guide.
\end{abstract}

\section{Introduction}

New Nuclide Guide-4 has been developed as a revised and updated version of the Nuclide Guide-3 [1]. It contains information on nuclear and decay characteristics of more than 3000 isotopes of 118 chemical elements. The information from such databases is widely used in calculations of induced activation, transmutation of isotopes, heat production. It is of special importance in planning experiments and processing the results of experiments. The data from the Nuclide Guide-4 was used for development of new Chart of Nuclides. The Guide and the Chart are intended for being used by students, engineers, scientists.

The necessity in revision of existing Nuclide Guides and Charts of nuclides is induced by performing new precise measurements and disagreement between the evaluated values in basic data libraries. For example, in tables 1-3 the evaluated data from different libraries are presented.

\section{Contents of the Nuclide Guide and the Chart of Nuclides}

In Nuclide Guide-4 for each nuclide nine nuclear and decay characteristics are given. In particular, spin, parity, mass of nuclide, magnetic moment (if available), mass excess, halflife or abundance, decay modes, branching ratios, emitted particles, energies of most intense gamma-rays and their intensities, decay energies and mean values of radiation energy per decay are presented. For stable and natural long-lived nuclides cross-sections of thermal neutron induced activation are indicated. The information presented in the Guide was compiled from 5 sources: 1) ENSDF [2], 2) atomic mass evaluation-2003 by Audi and Wapstra [5], 3) interactive

${ }^{a}$ Presenting author, e-mail: gol@ainf.rua
Table 1. Decay modes and branching ratios (in percent) for some isotopes from the ENSDF [2], NUBASE [3] and Nuclear Wallet Cards [4].

\begin{tabular}{cccc}
\hline isotopes & ENSDF & NUBASE & NWC \\
\hline Be-14 & $\beta^{-} 100$ & $\beta^{-} 100$ & $\beta^{-} 100$ \\
& $\beta^{-}$n 81(4) & $\beta^{-}$n 98(2) & $\beta^{-} \mathrm{n} 94$ \\
& $\beta^{-}$2n 5(2) & $\beta^{-} 2 \mathrm{n} 0.8(8)$ & $\beta^{-} 2 \mathrm{n} 6$ \\
& & & \\
B-7 & $\mathrm{p} ? \alpha ?$ & $\mathrm{p}$ & $\mathrm{p}, \alpha$ \\
C-8 & $\mathrm{p} 100, \alpha ?$ & $2 \mathrm{p}$ & $\mathrm{p} 100, \alpha ?$ \\
V-61 & - & $\beta^{-} 100, \beta^{-} \mathrm{n}<6$ & $\beta^{-} 100$ \\
Mn-64 & $\beta^{-} 100, \beta^{-} \mathrm{n} 33(2)$ & $\beta^{-} 100, \beta^{-} \mathrm{n} ?$ & $\beta^{-} 100, \beta^{-} \mathrm{n} 1.42$ \\
\hline
\end{tabular}

Table 2. Half-lives of some isotopes from the ENSDF [2], NUBASE [3] and Nuclear Wallet Cards [4].

\begin{tabular}{cccc}
\hline isotopes & ENSDF & NUBASE & NWC \\
\hline Ti-55 & $0.52(12) \mathrm{s}$ & $0.49(9) \mathrm{s}$ & $1.3(1) \mathrm{s}$ \\
Ti-56 & $0.15(3) \mathrm{s}$ & $0.164(24) \mathrm{s}$ & $0.200(5) \mathrm{s}$ \\
Ar-47 & $\approx 700 \mathrm{~ms}$ & $580(120) \mathrm{ms}$ & $1.23(3) \mathrm{ms}$ \\
Cr-60 & $0.49(1) \mathrm{s}$ & $0.56(6) \mathrm{s}$ & $0.57(6) \mathrm{s}$ \\
Cr-62 & $0.19(3) \mathrm{s}$ & $0.199(9) \mathrm{s}$ & $0.209(12) \mathrm{s}$ \\
Mn-46 & $41(+7-6) \mathrm{ms}$ & $37(3) \mathrm{ms}$ & $34(+5-4) \mathrm{ms}$ \\
\hline
\end{tabular}

data bases at web-sites www.nndc.bnl.gov, www.nucleide.org, 4) original evaluations of authors, 5) recent publications. Particularly, masses of nuclides were extracted from the file nuclear_masses03_rounded.txt [5]; mass excess for nuclides in their ground- and isomeric-states was calculated by authors on the basis of information stored in the file nuclear_masses03_original.txt [5]. The same information was used for calculation of decay energies. For some nuclides there are essential differences (especially for uncertainties) between the decay energies and their uncertainties calculated by authors and the data presented in NUBASE [3]. As other result of these calculations some decay modes indicated in 
Table 3. Spins and parities for some isotopes from the ENSDF [2], NUBASE [3] and Nuclear Wallet Cards [4].

\begin{tabular}{cccc}
\hline isotopes & ENSDF & NUBASE & NWC \\
\hline V-58 & - & $\left(3^{+}\right)$ & $\left(1^{+}\right)$ \\
V-61 & - & $\left(7 / 2^{-}\right)$ & $\left(3 / 2^{-}\right)$ \\
Fe-67 & $\left(5 / 2^{+}\right)$ & $\left(1 / 2^{-}\right)$ & \\
\hline
\end{tabular}

evaluated data libraries as possible were rejected since those are energetically prohibited. It concerns decay modes $\mathrm{p}, \alpha$ for Be-6 [4], $\alpha$ for B-7 [4], 2p? for Ge-60 [4]. Average energies of the radiation were calculated on the basis of information about single $\gamma$-lines which is stored in interactive data bases at web-site www.nndc.bnl.gov. Energies of most intensive $\gamma$-rays and their intensities are taken from the data bases at web-sites www.nndc.bnl.gov and www.nucleide.org. Some nuclear and decay characteristics for which experimental information is absent were calculated with using the systematics. These values are bracketed in the Guide.

Almost all the data is presented with uncertainties which were calculated by authors or extracted from data bases ENSDF [2] and NUBASE [3]. Sometimes the uncertainties for half-lives are given asymmetrically. In these cases we have used a procedure described in [3] to get more justified central value and its uncertainty.

The nuclear and decay characteristics presented in the Nuclide Guide- 4 were also inserted in the Chart of Nuclides. Two numbers (charge of the nucleus $\mathrm{Z}$ and number of neutrons N) define the nuclide place (its "box") in the Chart of Nuclides. The number of neutrons increases horizontally, the number of protons increases vertically. Thus, since the number of protons $\mathrm{Z}$ is simultaneously the serial number of a chemical element in periodic system, the horizontal sequence of "nuclide boxes" in the chart of nuclides corresponds to isotopes of the same chemical element. The vertical sequences of boxes define isotones, i.e., the nuclides having the same number of neutrons in the nucleus. The nuclides having the same mass number $\mathrm{A}$ (isobars) are positioned along the diagonals (from left lower corner to the right upper one). Along these lines the nuclear transformations of the nuclides are carried out by means of beta-decay and reactions $(p, n)$, (n,p) following the replacement of a neutron with proton, and vice versa. Directions of other kinds of decay and nuclear reactions are shown in the explanatory part of the chart of nuclides.

The appropriate nuclide boxes of the chart contain information of the principal nuclide characteristics. The symbol of a chemical element and mass number is specified in the first line inside the box for any nuclide. Values of mass excess (in $\mathrm{MeV}$ ) are presented in the second line. The quantum characteristics of nucleus in the ground state - its spin and parity, are presented in the third line. If a nuclide is characterized by metastable excited states having the life time exceeding $1 \mathrm{~ms}$ the isomer characteristics are specified in the same box. For this purpose the nuclide box is divided into two parts if one isomer is available, and into three parts if two isomers are available. Radionuclide half-life is presented in the fourth line as one of the most important nuclear decay characteristics of radioactive nuclides. In case of stable isotopes this line contains the nuclide percentage in natural mixture of the

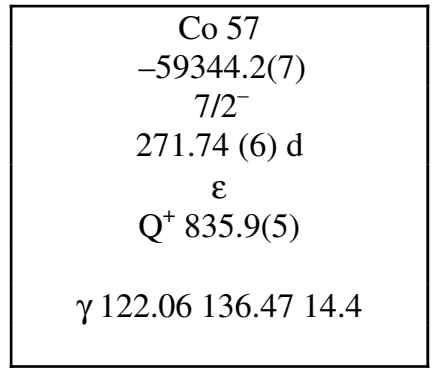

Fig. 1. Information box for ${ }^{57} \mathrm{Co}$.
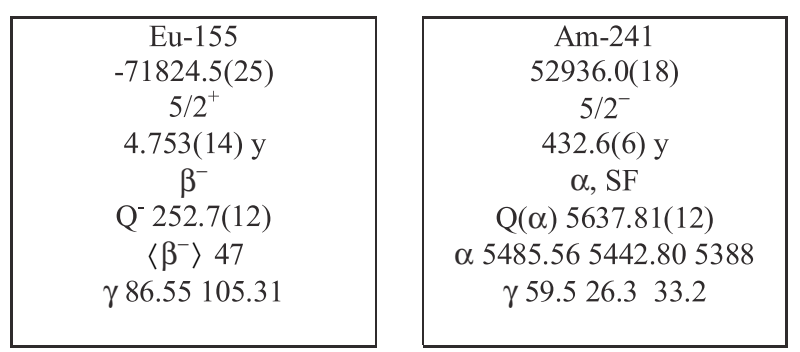

Fig. 2. Information boxes for ${ }^{155} \mathrm{Eu}$ and ${ }^{241} \mathrm{Am}$.
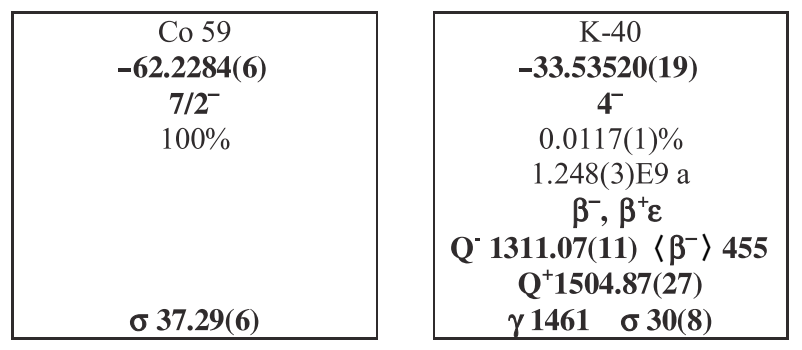

Fig. 3. Information boxes for ${ }^{59} \mathrm{Co}$ and ${ }^{40} \mathrm{~K}$.

chemical element under consideration. The fifth line contains the modes of nuclear transformations (decays) $\left(\alpha, \beta^{-}, \varepsilon, \varepsilon \beta^{+}\right.$, $\beta$, n, etc.) of the radioactive nuclides. Radiation capture cross section of thermal neutrons (activation cross section) in barns is presented in this line for the stable and natural long-lived nuclides.

Except for the specified principal characteristics located in 5 lines, the remaining 4 lines of boxes contain the additional nuclear decay characteristics of the radioactive nuclides as follows: total decay energy $\mathrm{Q}$ in $\mathrm{keV}$, average energy of betaradiation in the same units (bracketed), the energy of the most intensive alpha- and gamma-radiation (in keV). Mean radiation energy per decay $\langle\mathrm{R}\rangle$ is a quantitative characteristic indicating the contribution of the given radiation type to the energy $(Q)$ released in the decay.

The uncertainties of the recommended values are parenthetical and provided with the number of units of the last significant digit of the value: for instance, 40.1(22) means $40.1 \pm 2.2$

Below the examples of the boxes for ${ }^{57} \mathrm{Co},{ }^{155} \mathrm{Eu}$ and ${ }^{241} \mathrm{Am}$ are presented in figure 1 and figure 2.

As for the stable nuclides, the abundance of nuclide in natural mixture of isotopes (percentage) is indicated in the 
forth line and the thermal neutron activation cross section is indicated in the last line. The examples of the stable and natural unstable nuclide information boxes for ${ }^{59} \mathrm{Co}$ and ${ }^{40} \mathrm{~K}$ are given in figure 3 .

As to history, the necessity to develop the international charts of nuclides was discussed in 1994 at International Conference on Nuclear Data for Science and Technology, Gatlinburg, the USA. IAEA international working group had confirmed that there is a necessity to develop the international charts of nuclides. Opinion of more than 200 respondents from national and international organizations as a result of 1994 - 1996 attitude survey was the reason for developing the international charts of nuclides.

\section{References}

1. T.V. Golashvili, V.P. Chechev, O.O. Patarakin, N.E. Yakovlev, V.M. Kupriyanov, Zhao Zhixiang, Huang Xiaolong, Ge Zhigang, Zhou Chunmei, Nuclide Guide-3 (Atomic Energy Press, BeijingMoscow, 2004).

2. T.W. Burrows, Nucl. Instrum. Meth. 286, 595 (1990); http:/www.nndc.bnl.gov/

3. G. Audi, O. Bersillon, J. Blachot, A.H. Wapstra, Nucl. Phys. A 729, 3 (2003).

4. J.K.Tuli, Nuclear Wallet Cards, 7th edn. (Brookhaven National Laboratory, 2005); http:/www.nndc.bnl.gov

5. G. Audi, A.H. Wapstra, C. Thibault, Nucl. Phys. A 729, 337 (2003). 